\title{
The effects of lesions in amygdala or dorsomedial frontal cortex on reinforcement omission and noncontingent reinforcement in rats
}

\author{
JOHN H. McDONOUGH, JR. \\ Neuropsychology Branch, Biomedical Laboratory, Chemical Systems Laboratories \\ Aberdeen Proving Ground, Maryland 21010 \\ and \\ FREDERICK J. MANNING \\ U.S. Army Medical Research Unit-Europe, U.S. Army Medical Command Europe \\ Heidelberg, Federal Republic of Germany, APO New York 09102
}

\begin{abstract}
Two groups of six rats with lesions of the amygdala or dorsomedial frontal cortex were compared to normal controls in their response to occasional omission of food reward following training on an FI 60-sec schedule (Experiment 1). Both brain-damaged groups showed reliably greater increases in their responding in intervals following reward omission compared to their responding in intervals following reward delivery than did controls. In a second experiment, there was no difference between these groups in their response to complete elimination of reward (extinction). However, both groups with lesions reliably reduced responding to a greater extent than did controls when rewards were delivered noncontingently (free) on an FI 60-sec schedule. The results of both experiments were interpreted as demonstrating a reduced efficacy of secondary reinforcers to control the behavior of subjects with these limbic lesions.
\end{abstract}

Rats with lesions in the amygdala or dorsomedial frontal cortex may be impaired in their responses to the aversive effects of nonreward (Glass, Ison, \& Thomas, 1969; Gurowitz, Rosen, \& Tessel, 1970; Henke, 1973; Henke \& Maxwell, 1973). Rats with lesions of the amygdala do not change running speeds following shifts in reward magnitude in runway situations (Henke \& Maxwell, 1973; Kemble \& Beckman, 1970). Qualitatively similar results have been obtained in operant studies of rats with lesions in the amygdala using a behavioral contrast procedure in which one component of a mixed or multiple schedule is switched to extinction (Henke, Allen, \& Davison, 1972) or when reinforcement is occasionally omitted on a conventional fixed interval (FI) schedule (Henke, 1973). Rats with lesions of the dorsomedial frontal cortex are deficient in reversing a spatial discrimination habit (Stutz \& Rocklin, 1968), are impaired in the acquisition and retention of singlealternation learning (Barker \& Thomas, 1965), and fail to change running speeds in a runway when reward magnitudes are shifted (Gurowitz et al., 1970).

This work was performed primarily at the Department of Experimental Psychology, Division of Neuropsychiatry, Walter Reed Army Institute of Research, Washington, D.C. 20012.
These results have often been explained as the result of a lesion-produced reduction of a frustrative emotionality which is typically seen as an energizing effect on behavior following nonreinforcement.

However, the above results also could be, and have been, explained by a disruption of response inhibition, expressed as an increased tendency to perseverate in previous reinforced responses when reinforcement contingencies are changed. The failure of rats with amygdala lesions to alter running speeds in response to shifts in the magnitude of reward in a runway task was attributed to response perseveration (Kemble \& Beckman, 1970). In addition, rats with amygdala damage are impaired on acquisition of passive avoidance, efficient response patterning on a differential reinforcement of low rates (DRL) schedule, and noncued spatial alternation (Pellegrino, 1968). Likewise, Barker (1967) reported that rats with lesions of dorsomedial frontal cortex were severely impaired in their ability to perform a simple or complex sequential leverpress task, and that perseverative errors were evidenced even when the correct response sequence was cued with visual stimuli.

The present study tested these two theoretical positions using operant techniques by comparing the performance of brain-damaged rats with that of nor- 
mal controls. Experiment 1 used an operant analog of the double runway "frustration" task (Amsel \& Roussel, 1952), in which rats that have been trained on a conventional FI schedule of reinforcement are tested by occasionally omitting reward at the end of an interval (Staddon \& Innis, 1969). Under such conditions, normal rats increase their rate of responding in intervals following reward omission in a manner analogous to the increase in running speeds seen following omission of reward in the first goalbox of a double runway. In these circumstances, it would seem that the "lack of frustrative emotionality" theory would predict a smaller, or at least slower, increase in the response rates of subjects with lesions. However, "response perseveration" theory would seem to predict elevated responding following reward omission, since subjects are typically responding at high rates at the end of the fixed interval. Experiment 2 asked whether brain-damaged rats perseverate in circumstances where declines are produced in the response rates of intact rats, by either complete omission of reward from the schedule or by omission of the response requirement while still maintaining the schedule of reward. Under such conditions, the "lack of frustrative emotionality" theory would predict a slower than normal rate of decline in responding for subjects with lesions when reward is eliminated, but not when the response requirement is omitted, while a "response perseveration" deficit would predict a slower than normal decline in responding by subjects with lesions under both conditions.

\section{METHOD}

Subjects.

Twenty-four adult male albino rats of the Walter Reed strain [Wistar derived Wrm: WRC (WI) BR] served as subjects. ${ }^{1}$ Twelve rats served as unoperated controls, while groups of six rats each were administered bilateral stereotaxically placed radio-frequency lesions of the amygdala or the dorsomedial frontal cortex.

\section{Surgery and Histology}

Each experimental rat was anesthetized with sodium pentobarbital $(60 \mathrm{mg} / \mathrm{kg})$ IP and given $.1 \mathrm{cc}$ of atropine sulfate to facilitate respiration. The lesion electrode was an insect pin (No. 000), insulated except for $1.00 \mathrm{~mm}$ at the tip, and the ground electrode was attached to the scalp incision with a miniature alligator clip. Lesions were produced with a Grass Model LM3 radio-frequency lesion maker, with the intensity setting on 58 for a duration of $30 \mathrm{sec}$. All lesions were made with the skull level between lambda and bregma. Coordinates for the amygdala lesion were: P $2.0 \mathrm{~mm}$ from bregma, $\mathrm{L} 4.5 \mathrm{~mm}$ from the midline, and V $8.4 \mathrm{~mm}$ from the surface of the cortex. The dorsomedial frontal cortex lesion was produced by a series of lesions at the following coordinates: A 6.0, 4.5, 3.0, 1.5, and $0.0 \mathrm{~mm}$ from bregma, $\mathrm{L} 1.0 \mathrm{~mm}$ from the midline, and $\mathrm{V} 1.0$ and $2.0 \mathrm{~mm}$ from the surface of the cortex at each A-P point. All operated subjccts received ad-lib access to food and water for 2 weeks post - op, prior to the start of behavioral testing.

At the completion of the experiment, all subjects were killed with pentobarbital and perfused intracardially with saline, followed by $10 \%$ Formalin. The brains were then blocked and imbedded in paraffin. Coronal sections were cut, $40 \mu$ thick, and every
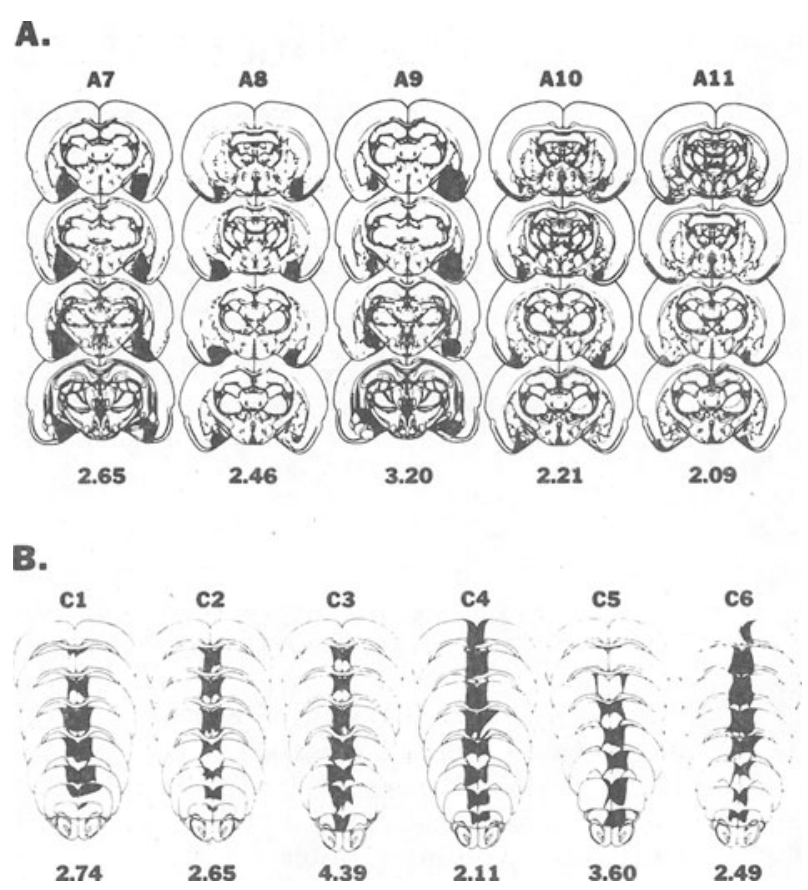

Figure 1. Reconstructions of the amygdala lesions (A), and dorsomedial frontal cortex lesions (B), on line drawings after König and Klippel (1963). The number under each reconstruction is that subject's mean omission ratio for $P\left(S^{R}\right)=.80$.

fourth section stained with cresyl violet. The histology from one amygdala subject was lost in processing. Figure 1 displays reconstructions of the lesions for both groups on outline drawings from König and Klippel (1963).

The amygdala lesions ranged from near complete destruction of all but the most rostral and most caudal portions to quite limited damage in the central portions of the cortical, basomedial, and basolateral nuclei. Of the various amygdaloid nuclei, the lateral was, by and large, the least damaged, while portions of the optic and lateral olfactory tracts were the main recipients of extra-amygdaloid damage.

Dorsomedial frontal cortex lesions were all confined to dorsal midline cortex, from the pole to just posterior of the level of the anterior commissure, with some slight damage to the corpus callosum. The most heavily damaged area corresponds to the "shoulder region" of the dorsomedial frontal cortex, as described by Leonard (1969).

\section{Apparatus}

Behavioral testing was conducted in two identical BRS-Foringer two-lever operant conditioning chambers for rats. Only the left lever was used in the study. A single white pilot light over this lever and a 6-W, fluorescent houselight were illuminated throughout the experimental session, except at the times noted below. White noise masked extraneous sounds while these lights were on. Programming and data recording were accomplished with solidstate logic modules, Gerbrands cumulative recorders, and electromechanical counters.

\section{Procedure}

Experiment 1. Following recovery from surgery, the body weights of all subjects were reduced and maintained at $80 \%$ of ad-lib levels for the duration of the experiment. The subjects were then randomly assigned to either chamber 1 or chamber 2 and, after 
magazine training, were trained to leverpress for $45-\mathrm{mg}$ Noyes food pellets. All subjects were then given five 30-min sessions on $\mathrm{CRF}$, followed by 15 sessions on a FI 60 -sec schedule. Each reinforcement during these sessions and throughout the remainder of the experiment consisted of the delivery of three food pellets. Pellet delivery was accompanied by the offset of the houselight, pilot light, and white noise for $1.5 \mathrm{sec}$. A second pilot light, over the inoperable right lever, was illuminated during this time. Sessions ended with the 40th reinforcement. These 15 sessions, where the probability of reinforcement $\left[\mathrm{P}\left(\mathrm{S}^{\mathrm{R}}\right)\right]$ was unity, were followed by 15 sessions with the $\mathrm{P}\left(\mathrm{S}^{R}\right)$ at the end of a given interval was .80 , and an additional 15 sessions with $\mathrm{P}\left(\mathrm{S}^{\mathrm{R}}\right)=.50$. Regardless of whether pellets were delivered or omitted, the first response after the end of the 60 -sec interval produced all the secondary stimuli previously associated with pellet delivery, i.e., offset of houselight, left pilot light, and white noise and onset of right pilot light for $1.5 \mathrm{sec}$. Responses in the intervals following pellet omission were recorded separately from those following pellet delivery, and in both cases responses in the 1st, 2 nd, 3rd and 4th quarters of the FI were recorded separately.

Experiment 2. At the completion of Experiment 1, each group was divided into two subgroups matched as evenly as possible on total responses over the last three sessions. One subgroup from each of the groups with lesions and one subgroup from each of the normal control groups were then placed on extinction $\left[\mathrm{P}\left(\mathrm{S}^{\mathrm{R}}\right)\right.$ $=0.00]$. The remaining rats were placed on a noncontingent (FREE) schedule of reinforcement. The FREE schedule delivered pellets automatically at the end of the 60 -sec interval rather than contingent on a leverpress as with the conventional FI schedule. Experiment 2 terminated when each subject had been run for 15 sessions with one of these new reinforcement contingencies (EXT or FREE) in effect.

\section{RESULTS}

\section{Experiment 1}

A one-way ANOVA of the mean number of responses over the last five baseline sessions of the FI 60 -sec schedule revealed no reliable group differences $[F(2,21)=.70, p>.10]$, although the normals averaged 533 responses, the amygdala group, 431, and the dorsomedial frontal cortex group, 377. A second measure of FI performance, interval half-life, was interpolated for each animal by graphing the cumulative percentage of responses within quartiles of the intervals vs. time and then dropping a line from where the function crossed $y=$ $50 \%$. This measure is a quantitative index of the "scalloping" of responses within the fixed intervals. Response half-lives were calculated and averaged for each animal over the last five FI 60 -sec baseline sessions and analyzed with a one-way ANOVA. The analysis showed no differences between groups in interval half-life $[\mathrm{F}(2,21)=1.51, \mathrm{p}>.10]$. The interval half-lives for the three groups were: normals, $45.6 \mathrm{sec}$; amygdalas, $49.8 \mathrm{sec}$; and dorsomedial frontal cortex, $51.3 \mathrm{sec}$. There were, thus, no reliable differences between the normals and the groups with lesions in their quantitative or qualitative performance on the final five baseline FI 60 -sec sessions.

When the probability of food-pellet delivery at the end of a given interval was changed from $P\left(S^{R}\right)$ $=1.00$ to $\mathrm{P}(\mathrm{SR})=.80$, there was a marked in- crease in the rate of responding in intervals following pellet omission relative to the rates observed in intervals following pellet delivery. This effect is illustrated for all groups in Figure 2 where group mean "omission ratios" are plotted in blocks of three sessions. The "omission ratios" were derived by dividing the mean number of responses in intervals following pellet omission by the mean number of responses following pellet delivery. Thus, ratios greater than 1.00 indicate increased responding in the intervals following pellet omission. The left portion of Figure 2 shows the data for the 15 sessions with $\mathrm{P}(\mathrm{SR})=.80$, and the right side shows the 15 sessions with $\mathrm{P}(\mathrm{SR})=.50$. These data were analyzed separately with multifactor ANOVAs with blocks of sessions as a repeated measure (Winer, 1971, p. 601). For the 15 sessions with $\mathrm{P}(\mathrm{SR})$ $=.80$, the analysis revealed reliable effects for lesion $(\mathrm{F}(2,21)=7.16, \mathrm{p}<.01]$ and the Lesion by Sessions interaction $[\mathrm{F}(8,84)=2.74, \mathrm{p}<.01]$, while the sessions effect was not reliable $[\mathrm{F}(4,84)=1.39]$. A Newman-Keuls test of ordered means showed that both groups with lesions displayed a greater increase in responding to pellet omission than did the normals $(p<.01)$, and that the dorsomedial frontal cortex group was more responsive to pellet omission than the amygdalas $(p<.01)$. The Lesion by Sessions interaction was clearly due to the extremely high omission ratios displayed by the dorsomedial frontal cortex group in the first block of sessions with $\mathrm{P}(\mathrm{SR})$ $=.80$. This large increase in responding following pellet omission then slowly delcined over trials such that by the final block of sessions there was no difference between the dorsomedial frontal cortex group and the amygdala group on the omission ratio measure.

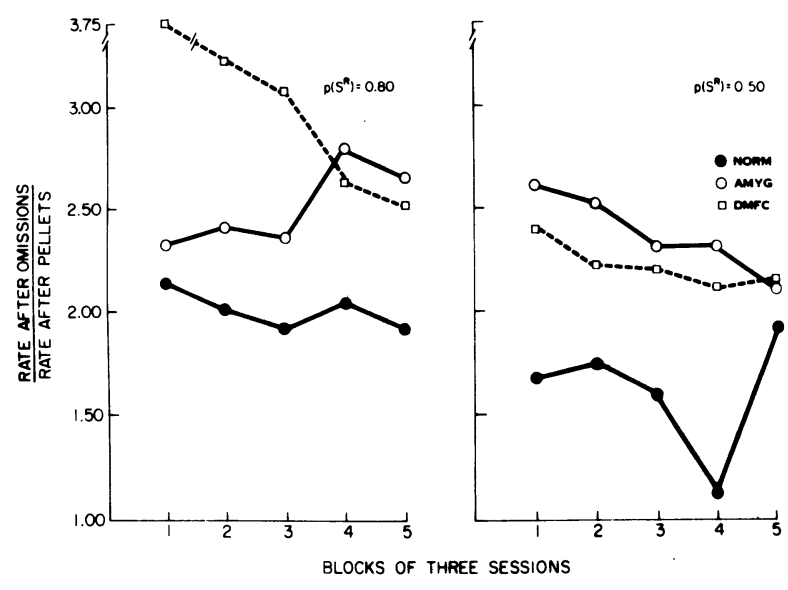

Figure 2. The mean ratio of response rate in intervals immediately following pellet omission to response rate in intervals following pellet delivery, for normal controls and the two lesion groups, plotted in blocks of three sessions. The left panel summarizes data from the 15 sessions where probability of reward at the end of an interval was .80 ; the right panel, the 15 sessions where this probability was $\mathbf{. 5 0}$. 
Analysis of similar data with $\mathrm{P}\left(\mathrm{S}^{\mathrm{R}}\right)=.50$ revealed no reliable effects for groups $[\mathrm{F}(2,21)=2.39$, $\mathrm{p}<.10]$, sessions $[\mathrm{F}(4,84)=2.03, \mathrm{p}<.10]$, or Groups by Sessions $[F(8,84)=1.14, p<.10]$. While the analysis failed to show any statistically reliable effects, it should be noted that the data fall into the same general pattern as seen with $\mathrm{P}(\mathrm{SR})=.80$, viz, both amygdala and dorsomedial frontal cortex groups showing a larger omission effect than normals.

One notable aspect of the data that is not readily apparent concerns the relationship between baseline FI performance and subsequent response to pellet omission. Comparison of group mean response rates with the group omission ratios that are plotted in Figure 2 suggests no simple prediction of the latter from the former. However, within a given group, the correlation between mean total responses in Baseline Sessions 11-15 and the mean omission ratio for the 15 sessions $\mathrm{P}(\mathrm{SR})=.80$ are all high and negative (normals, $\mathrm{r}=-.80$; amygdala, $\mathrm{r}=-.54$; dorsomedial frontal cortex, $r=-.66$ ). Thus, within a group, the lower the baseline rate of responding, the more marked the subsequent response to pellet omission (i.e., the higher the omission ratio). This finding suggests that the increase in rate of response in intervals following pellet omission is likely to be a qualitatively similar phenomenon for both braindamaged and normal animals.

Spearman rank difference correlations (Guilford, 1965 , p. 305 ) were computed between lesion size and mean omission ratios for $\mathbf{P}(\mathrm{SR})=.80$. Two colleagues who were blind as to the results of the study were asked to consensually rank each lesioned group as to magnitude of destruction. Also, in the case of the dorsomedial frontal cortex group, they were asked to rank the lesions on anterior/posterior placement. The correlation between lesion size and omission ratios for the amygdala group was $\varrho=.90$ $(\mathrm{N}=5 ; \mathrm{p}<.05)$, while that for the dorsomedial frontal cortex group was $\varrho=.66(\mathrm{~N}=6 ; \mathrm{p}>$ .10). The correlation between anterior/posterior extent of the dorsomedial frontal cortex lesions and omission ratios was $\varrho=-.89(\mathrm{~N}=6 ; \mathrm{p}<.05)$. Thus, the greater the size of the amygdala lesions, the greater the magnitude of the omission ratio, while for the dorsomedial frontal cortex group, the more anteriorally placed lesions were associated with higher omission ratios. Correlations were also computed between lesion size and mean total responses in Baseline Sessions 11-15. The results suggested no relationship between lesion size and baseline response rate for either the amygdala $(\varrho=.30)$ or the dorsomedial frontal cortex $(\varrho=.32)$ group.

\section{Experiment 2}

A one-way ANOVA was performed on the total number of responses emitted in intervals following reward for the three groups on the last day $\mathrm{P}(\mathrm{SR})$ $=.50$. The results revealed no reliable differences between groups on this measure $[\mathrm{F}(2,21)=1.33]$. Therefore, all groups were responding at equivalent levels following pellet deliveries prior to the start of Experiment 2.

Elimination of reinforcements (EXT) and elimination of the response requirement (FREE) both produced declines in the responding of all groups. Figure 3 illustrates the reduction in relative rates of responding produced by these two procedures. Relative rate of response is the rate of responding as a proportion of the rate of rewarded responses in the session preceding the first EXT or FREE session. Repeated-measures ANOVAs of these data both indicated reliable sessions effects [FREE: $\mathrm{F}(4,32)=9.85, \mathrm{p}<.01 ; \mathrm{EXT}: \mathrm{F}(4,32)=51.94$, $\mathrm{p}<.01$ ), which reflect the decline in responding produced by both procedures. In addition, the analysis indicated a reliable lesion effect for the FREE procedure $[F(2,8)=9.04, p<.01]$. A NewmanKeuls test of this effect showed that both groups with lesions had a greater decline in responding over the 15 FREE sessions than the normal controls. The analysis revealed no reliable group effects for the EXT procedure $[F(2,8)=1.91]$, and in neither analysis were the Group by Sessions interactions reliable [FREE: $F(8,32)=1.03$; EXT: $F(8,32)=1.36]$.

\section{DISCUSSION}

The results of Experiment 1 show that rats with lesions of the amygdala or dorsomedial frontal cortex are more responsive than normal rats to occasional omission of reward while working on an FI

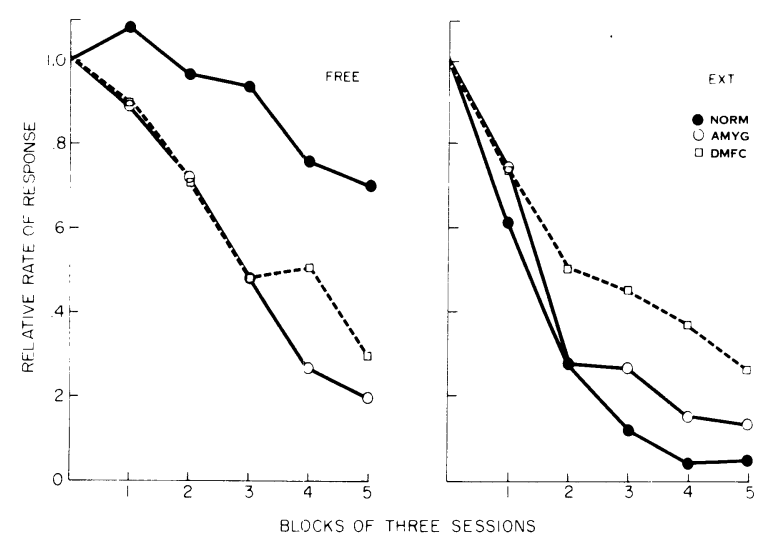

Figure 3. The mean ratio of response rate under noncontingent reinforcement (FREE) or extinction (EXT) to response rate on the day preceding the introduction of these conditions, for the normal controls and the two lesion groups, plotted over blocks of three sessions. 
schedule. The magnitude of the omission effect in these brain-damaged subjects was shown to be related to the extent of amygdala damage and to the location of the dorsomedial frontal cortex lesions. The finding that normal rats double their number of responses in intervals following pellet omission agrees with the results of previous studies that have subjected intact rats to similar procedures (Staddon \& Innis, 1969; Zimmerman, 1971). However, the exaggerated response to pellet omission displayed by both lesion groups is not consistent with the results and interpretations of a number of previously reported studies. Rats with lesions of the dorsomedial frontal cortex showed a smaller "depression effect" than normals when reward magnitude in a runway task was shifted from 15 pellets to 1 (Gurowitz et al., 1970). Likewise, rats with lesions of the amygdala failed to alter running speeds in a runway when reward magnitude was either decreased (5 pellets to 1 pellet) or increased (1 pellet to 10 pellets) (Kemble \& Beckman, 1970), or when reinforcement was omitted from the first goalbox of a double runway (Henke \& Maxwell, 1973). Also, Henke (1973) reported that rats with lesions of the amygdala trained to respond on an FI 2-min schedule for water reinforcement failed to increase response rates in intervals following occasional omission of reward. These failures of subjects with lesions to modify their behavior in response to reductions or omission of reward have been interpreted as reflecting a "lack of frustrative emotionality." While the subjects with dorsomedial frontal cortex or amygdala damage in this study did not respond like normals to pellet omission, their elevated response rates can hardly be attributed to a "lack of frustrative emotionality," at least if this concept is defined as elevated responding in intervals following reward omission.

Some theoretical discussion of the "omission effect" seen on interval schedules have tended to emphasize the discriminative effects of reward or reward omission rather than the motivational consequences (Staddon, 1967, 1970; Staddon \& Innis, 1969). Analysis of leverpress data in the intervals following reward omission showed that the increase in responding in these intervals was due to a shortening of the pause commonly observed following the presentation of the primary and/or secondary reinforcers, rather than an increase in the "running" response rate (Staddon \& Innis, 1969). In this sense, presentation of the secondary stimuli presumably conditioned to reward delivery at the end of an interval produces less inhibition of responding in the subsequent interval than does reward plus associated secondary stimuli. The greater responding of the brain-damaged subjects observed in response to reward omission may thus be due to the failure of these associated secondary stimuli to inhibit subsequent responding to the degree that it does in normal subjects, even though the ability of primary reinforcement to inhibit responding remained unimpaired. This explanation would be consistent with, if not easily predictable from, hypotheses of dorsomedial frontal cortex and amygdala function which stress their role in control of response inhibition (or the converse, perseveration) (Barker, 1967; Glass et al., 1969; Kemble \& Beckman, 1970; Pellegrino, 1968; Slotnick \& McMullen, 1973).

However, an increased tendency to perseverate due to a disruption of response inhibition cannot serve as a unifying concept for the results of both experiments. Experiment 2 clearly shows that the animals with lesions did not continue to respond any longer than normal controls when reward was completely omitted, and they reduced responding to levels even lower than normals in the FREE procedure. The finding of no differences between the reduction or responding by normals and by subjects with lesions subjected to extinction is consistent with the data from other operant studies of rats with amygdala or dorsomedial frontal cortex lesions that show no effect on extinction following training on a variety of operant schedules (Barker, 1967; Henke, 1972, 1973; Henke, Allen \& Davison, 1972; Henke \& Bunnell, 1971; Pellegrino, 1968). However, Kemble and Beckman (1970) did report that rats with lesions of the amygdala were slower than normals to extinguish a runway response, which points out the difficulty in equating results from runway tasks with those obtained in leverpress situations. No previous research has related the effect of these two types of lesions to reductions of responding produced by the FREE procedure of Experiment 2 . Nevertheless, the faster and greater reduction in responding of the lesioned subjects in this condition shows that they had no difficulty in adjusting their behavior to changes in reinforcement contingencies.

The finding of no reliable differences between groups in the extinction portion of Experiment 2 provides perhaps the strongest argument against either the "lack of frustrative emotionality" theory or the disruption of "response inhibition" theory, which have been advanced to explain amygdala or dorsomedial frontal cortex dysfunction. Frustrative emotionality due to nonreward has been posited to contribute significantly to the reductions in responding observed during behavioral extinction of a positively motivated response (Amsel, 1958, 1962). Likewise, behavioral extinction should provide a perfect situation for the manifestation of response inhibition. It must be concluded, then, that neither theory provides a parsimonious explanation of the results of these two experiments. 
However, the results the FREE portion of Experiment 2 do lend additional support to the interpretation that secondary reinforcers exert less stimulus control over the behavior of animals with these two types of lesions. When animals are switched from an FI schedule of reinforcement to a FREE schedule, continued responding may be assumed to be maintained by one of two means. First, since response rates are typically high near the terminal portion of a fixed interval, there is a high probability that responding would be adventitiously reinforced on a FREE schedule. However, this would be expected to occur across all groups and does not account for the differential declines in responding observed between the brain-damaged and normal groups. Second, continued responding could be maintained by secondary reinforcers which have become conditioned to reward delivery. The results of Experiment 1 indicate that secondary reinforcers may exert far less stimulus control over the behavior of animals with these lesions than they do over that of normal subjects when rewards are occasionally omitted. On the FREE schedule, these secondary reinforcers may again exert stronger control over the behavior of the normal subjects, resulting in greater response rates when compared to those of the animals with lesions. Conversely, it can be argued that the primary reinforcer-i.e., food delivery, exerts much stronger control over the subsequent behavior of the brain-damaged subjects in these experiments. Only when reward delivery was unpredictable $[\mathrm{P}(\mathrm{SR})=.80]$ or noncontingent (FREE) were there any reliable differences in responding between the subjects with lesions and the controls. When reward delivery was predictable $[\mathrm{P}(\mathrm{SR})=1.0]$ or completely eliminated (extinction), the brain-damaged animals were indistinguishable from normals.

\section{REFERENCES}

Amsel, A. The role of frustrative nonreward in noncontinuous reward situations. Psychological Bulletin, 1958, 55, 102-119.

AmSE L, A. Frustrative nonreward in partial reinforcement and discrimination learning: Some recent history and a theoretical extension. Psychological Review, 1962, 69, 306-328.

Amsel, A., \& Roussel, J. Motivational properties of frustration: 1. Effect on a running response of the addition of frustration to the motivational complex. Journal of Experimental Psychology, 1952, 43, 363-368.

BARKER, D. J. Alterations in sequential behavior of rats following ablations of midline limbic cortex. Journal of Comparative and Physiological Psychology, 1967, 64, 453-460.

Barker, D. J., \& Thomas, G. J. Ablation of cingulate cortex in rats impairs alternation learning and retention. Journal of Comparative and Physiological Psychology, 1965, 60, 353-359.
Glass, D. H., Ison, J. R., \& Thomas, G. J. Anterior limbic cortex and partial reinforcement effects on acquisition and extinction of a runway response in rats. Journal of Comparative and Physiological Psychology, 1969, 69, 17-24.

Guilford, J. P. Fundamental statistics in psychology and education. New York: McGraw-Hill, 1965.

Gurowitz, E. M., Rosen, A. J., \& Tessel, R. E. Incentive shift performance in cingulectomized rats. Journal of Comparative and Physiological Psychology, 1970, 70, 476-481.

Henke, P.G. Effects of reinforcement omission on rats with lesions of the amygdala. Journal of Comparative and Physiological Psychology, 1973, 84, 187-193.

Henke, P. G., Allen, J. D., \& Davison, C. Effects of lesions in the amygdala on behavioral contrast. Physiology \& Behavior, $1972,8,173-176$.

Henke, P. G., \& Burnell, B. N. Reinforcement and extinction interactions after limbic lesions in rats. Communications in Behavioral Biology, 1971, 6, 329-333.

Henke, P. G., \& Maxwell, D. Lesions in the amygdala and the frustration effect. Physiology \& Behavior, 1973, 10, 647-650.

Kemble, E. D., \& Beckman, G. J. Runway performance of rats following amygdaloid lesions. Physiology \& Behavior, 1970, $5,45-47$.

König, J. F. R., \& KLIPPEL, R. A. The rat brain. Baltimore: Williams \& Wilkins, 1963.

LeOnARD, C. M. The prefrontal cortex of the rat. 1. Cortical projections of the mediodorsal nucleus. II. Efferent connections. Brain Reserach, 1969, 12, 321-343.

Pellegrino, L. Amygdaloid lesions and behavioral inhibition in the rat. Journal of Comparative and Physiological Psychology, $1968,65,483-491$.

Slotnick, B. M., \& McMullen, M. F. Response inhibition deficits in mice with septal, amygdala, or cingulate cortical lesions. Physiology \& Behavior, 1973, 10, 385-389.

STADDon, J. E. R. Attention and temporal discrimination: Factors controlling responding under a cyclic-interval schedule. Journal of Experimental Analysis of Behavior, 1967, 10, 349-359.

Staddon, J. E. R. Temporal effects of reinforcement: A negative "frustration" effect. Learning and Motivation, 1970, 1, 227-247.

Staddon, J. E. R., \& INNIS, N. K. Reinforcement omission on fixed-interval schedules. Journal of the Experimental Analysis of Behavior, 1969, 12, 689-700.

STUtz, R. M. \& Rocklin, K. W. Cingulate and fornix lesions: Effects on two types of reversal learning. Journal of Comparative and Physiological Psychology, 1968, 65, 520-523.

Winer, B. J. Statistical principles in experimental design. New York: McGraw-Hill, 1971.

Zimmerman, D. W. Rate changes after unscheduled omission and presentation of reinforcement. Journal of the Experimental Analysis of Behavior, 1971, 15, 216-270.

\section{NOTE}

1. In conducting the research described in this paper, the investigators adhered to the "Guide for Laboratory Facilities and Care," as promulgated by the Committee on Revision of the Guide for Laboratory Facilities and Care of the Institute of Laboratory Animal Resources, National Academy of ScienceNational Research Council.

(Received for publication November 21, 1978; revision accepted February $9,1979$. 